\title{
Cobertura vacinal real do esquema básico para o primeiro ano de vida numa Unidade de Saúde da Família
}

\author{
Basic real immunization coverage for the first year of life in a Family Health Unit \\ La cobertura de vacunación del esquema básico real para el primer año \\ de vida en una Unidad de Salud de la Familia \\ Sandra Maria Magalhães Villela Carneiro ${ }^{\star *}$, Simone Schwartz Lessa ${ }^{2}$, João Alfredo Lins Guimarães ${ }^{3}$, \\ Marina Moraes Loepert ${ }^{4}$, Diego Barbosa da Silva ${ }^{5}$
}

Palavras-chave: Cobertura Vacinal Vacinação Programas de Imunização Esquema de Imunização.

\section{Resumo}

Objetivos: Avaliar a cobertura vacinal real do esquema básico para o primeiro ano de vida numa Unidade de Saúde da Família de Maceió-AL. Métodos: Estudo do tipo transversal com amostragem do tipo não probabilística no qual foi aplicado questionário através do responsável de todas as crianças maiores de 12 e menores de 24 meses. A carteira de vacinação foi utilizada como fonte de informação. Considerou-se completado o esquema básico, a criança que recebeu a dose das vacinas na idade e intervalos corretos. As variáveis categóricas foram dispostas em proporções. Foram calculados média e desvio padrão para as variáveis numéricas. As diferenças nas proporções das variáveis entre os grupos de crianças com CV adequada e não adequada foram medidas através do Teste do $\chi^{2}$ de Pearson\% ( $p<0,05)$. Resultados: Obteve-se uma amostra de 66 crianças, das quais, 24,2\% ( $n=16)$ completaram 0 esquema básico de vacinação. Foi observado que $63,6 \%$ das crianças haviam apresentado algum tipo de morbidade nos três meses que antecederam a entrevista. A média de número de moradores foi de $4,7 \pm 1,8$, enquanto que o número médio de irmãos foi de $1,0 \pm 1,0$. Verificou-se que $75,8 \%$ ( $n=50$ ) das crianças faziam parte das classes C2 ou D. A média de idade do responsável foi de $30,53 \pm 11$, variando de 16 a 62 anos. Conclusões: A cobertura para cada uma das vacinas foi sempre maior que a cobertura para 0 esquema completo. Verificou-se que parcela significativa das crianças desta pesquisa não recebeu todas as doses preconizadas, nas idades adequadas e com os intervalos corretos. Portanto, a unidade necessita aprimorar seus procedimentos técnicos e administrativos a fim de garantir a plenitude da cobertura vacinal em data oportuna.

Keywords:

Immunization Coverage

Vaccination

Immunization Programs Immunization Schedule.

\section{Abstract}

Purpose: to evaluate basic immunization coverage for the first year of life in a family health unit in Maceio, Alagoas, Brazil. Methods: it was a cross-sectional study. Sixty six children from 12 to 24 months old were included. All parents or answerable persons for the children undergone to an interview. Additional data were collected from immunization cards for each child. Basic immunization coverage was considered complete when children received immunization on adequate age and intervals. Categorical variables were disposed in proportions. Mean and standard deviation were calculated for numeral variables. Differences between appropriate and inappropriate vacinal coverage groups were measured through Pearson's $\chi^{2}(p<0.05)$. Results: We obtained a sample of 66 children, of whom $24,2 \%(n=16)$ completed the

\footnotetext{
Universidade Estadual de Ciências da Saúde de Alagoas (UNCISAL).smagalhaes25@hotmail.com

2 Universidade Estadual de Ciências da Saúde de Alagoas (UNCISAL). ss-lessa@uol.com.br

${ }^{3}$ Universidade Estadual de Ciências da Saúde de Alagoas (UNCISAL). joaolegista@globo.com

${ }^{4}$ Universidade Estadual de Ciências da Saúde de Alagoas (UNCISAL). mmoraes|@hotmail.com

${ }^{5}$ Universidade Estadual de Ciências da Saúde de Alagoas (UNCISAL). diego_valeouro@hotmail.com

*Autor correspondente.

Conflito de interesses: declaram não haver.

Fonte de financiamento: nenhuma.

Recebido em: 27/05/2011

Aprovado em: 23/05/2012
} 
basic immunization schedule. The average number of residents was $4,7 \pm 1,8$, while the averege number of siblngs was $1,0 \pm 1,0$. It was found that $75,8 \%(n=50)$ of children were part of the $C 2$ or D. The average age of the person responsible was $30,53 \pm 11$ ranging from 16 to 62 years. Conclusion: The coverage for each of vaccines was always greater than the coverage for theentire scheme. It was found that a significant portion of children in this research did not receive all recommended doses, at ages appropriate and corret intervals. Therefore, the init needs to improve it's technical procedures, and administrative in order to ensure full immunization coverage in due course.

Palabras clave:

Cobertura de Vacunación La Inmunización

Los Programas de Inmunización Esquema de Inmunización

\section{Resumen}

Objetivos: Evaluar la cobertura actual del régimen de la unidad básica de la vida en una familia de La Salud Maceió-AL. Métodos: Estudio transversal realizado en la Unidad de Salud de la Familia en Meceió Dr. Helvio Auto-AL. Se administró un cuestionario mediante entrevista a la persona responsable de todos los niños de 12 y menores de 24 meses. La tarjeta de vacunación se utilizó como fuente de información. Se consideró relación completa infantil básico que recibieron una dosis de la vacuna en intervalos de edad y correcta. Resultados: Se obtuvo una muestra de 66 niños. $24,2 \%$ ( $n=16$ ) niños completaron el esquema básico de inmunización. Se observo que 63,6\% de los niños había sufrido algún tipo de morbilidad em los três meses anteriores a La entrevista. El número médio de habitantes fue de 4,7 $\pm 1,8$, mientras que el número promedio de hermanos fue de 1,0 $\pm 1,0$. Se encontro que el $75,8 \%$ ( $n=50$ ) de los niños formaban parte de la C2 y D. El promedio de edad de La persona responsable fue 30,53 \pm 11 entre 16 y 62 años. Conclusiones: La cobertura de cada uma das vacunas fue siempre mayor que La cobertura para todo el sistema. Se encontro que uma porción significativa de los niños em estúdio no recibieron todas lãs dosis recomendadas, a intervalos de edad apropiada y correcta. Por lo tanto, La unidad tiene que mejorar SUS procedimientos técnicos y administrativos a fin de garantizar La cobertura de vacunacion completa em su debido momento.

\section{Introdução}

A cobertura vacinal $(\mathrm{CV})$ é um importante indicador de saúde das populaçóes e da qualidade da atençáo dispersada pelos serviços básicos de saúde ${ }^{1}$. Pode ser entendida como a proporção de crianças menores de um ano que receberam o esquema completo de vacinação em relação aos menores de um ano existentes na população, entendendo-se por esquema completo a aplicaçáo de todas as vacinas preconizadas pelo Programa Nacional de Imunizaçóes (PNI), cujas doses foram aplicadas nas idades corretas (adequação epidemiológica) e com intervalos corretos (adequaçáo imunológica) ${ }^{2}$. O estudo desse indicador, além de apontar aspectos da saúde infantil e da atuação dos serviços, subsidia o processo de planejamento, especialmente a reestruturação das açóes ${ }^{1}$. A CV no final dos anos 1980 ficava em torno dos 60\%, como média nacional, chegou, a partir da metade da década de 1990, a estimativas iguais ou superiores às preconizadas $-90 \%$ para a BCG e $95 \%$ para as demais vacinas ${ }^{3}$. O declínio acelerado de morbimortalidade por doenças imunopreveníveis nas décadas recentes, em nosso país e em escala mundial, serve de prova inconteste do enorme benefício que é oferecido às populaçóes por intermédio das vacinas ${ }^{4}$.

No Brasil as vacinas do esquema básico recomendadas para crianças menores de um ano são as seguintes: BCG - ID (dose única), vacina contra hepatite B (HB) (3 doses), vacina oral contra pólio (VOP) (3 doses), vacina tetravalente (DTP + Hib) (3 doses), vacina contra febre amarela (dose única) e vacina tríplice viral (SRC): sarampo, rubéola e caxumba (dose única) ${ }^{5}$. Em 2006, a vacina contra rotavírus foi incluída no Programa Nacional de Imunização ${ }^{6}$.
Em 2010 duas novas vacinas foram incluídas no calendário básico de vacinação disponível na rede pública de saúde: a pneumocócica 10-valente e a antimeningococo-C. A partir de 2011, elas farão parte do calendário básico de vacinação da criança específico para os menores de um ano ${ }^{7}$.

A escolha dos esquemas de vacinação tem como base os estudos realizados durante a fase de desenvolvimento das vacinas, sendo adotados os esquemas para os quais existem as melhores evidências de eficácia; portanto, recomenda-se seguir o mais fielmente possível as recomendaçóes para idade mínima de vacinação e intervalo entre as doses das vacinas ${ }^{8}$. A administração de vacinas muito precocemente e/ou com intervalos inferiores aos mínimos aconselhados pode diminuir a resposta imunológica e não devem ser consideradas válidas ${ }^{8}$. Nestas circunstâncias, deverá ser administrada uma nova dose, 4 semanas depois. Além disso, pode ainda aumentar o número de reaçóes adversas devido, provavelmente, à formaçáo de complexos antígeno-anticorpo ${ }^{8}$. Já o aumento do intervalo entre as doses não invalida as anteriores e, portanto, não exige que se reinicie o esquema com qualquer das vacinas do calendário 9 .

A vacinaçáo de rotina consiste no estabelecimento de um calendário nacional de vacinaçóes que deve ser aplicado a cada indivíduo a partir do seu nascimento, visando garantir, no âmbito individual, a prevenção específica das doenças imunopreveníveis; e, no âmbito coletivo, a indução da imunidade de massa, responsável pela interrupção da transmissão ${ }^{2}$. Para que o primeiro efeito se observe basta que cada criança vacinada torne-se uma criança imunizada; isto é, que a vacina seja aplicada em condiçôes que preservem sua eficácia e que a criança reúna as condições de saúde para 
desenvolver a imunidade assim induzida. Já para a obtenção do segundo efeito, será necessário que, além das condiçóes anteriormente mencionadas, a cobertura vacinal seja alta e homogênea ${ }^{2}$. O Ministério da Saúde (MS) preconiza os percentuais de cobertura vacinal de rotina como metas: 95\% de cobertura para o esquema básico de vacinação para menores de um ano de idade .

Para se alcançar e manter coberturas elevadas, um programa de vacinação tem de passar por avaliaçôes frequentes, nas quais determinados indicadores devem ser medidos utilizando-se instrumentos e informaçôes disponíveis nas comunidades ${ }^{4}$. A avaliação pode ser feita por meio de dois métodos. $\mathrm{O}$ primeiro é o método administrativo, estimado rotineiramente a partir das informaçóes dos boletins produzidos pelos serviços de saúde, ambulatórios e hospitais. Esse é o método mais utilizado devido à disponibilidade e facilidade de acesso dos dados. Entretanto, existe a possibilidade da CV assim avaliada náo refletir a cobertura real, pois as informaçóes sáo relativas às doses aplicadas, independentemente da idade e do intervalo entre as aplicações, acarretando o desconhecimento da permanência de indivíduos suscetíveis, além de que as doses aplicadas com intervalos mais curtos podem não desenvolver imunidade. Soma-se a isso o fato de que os dados de produçáo estấo sujeitos a erros de registro (sub-registro, duplicidade de dados) e não mostram algumas especificidades como informações sobre aquelas crianças que, embora residindo na área de influência do serviço de saúde, foram vacinadas em serviços de outras áreas ${ }^{10}$.

O segundo método de análise da cobertura vacinal é o método estatístico, obtido por meio de inquéritos populacionais, o qual apresenta vantagens, pois não sofre influência da invasão ou evasão de crianças. Permite também, quando programado, coletar as variáveis socioeconômicas da área de abrangência e das famílias, constatar a idade de administração de cada dose de vacina e o intervalo entre elas, obter informaçóes sobre as crianças que, embora residindo na área de influência do serviço de saúde, foram vacinadas em outros serviços e as que receberam vacinas em serviços não vinculados ao Sistema Único de Saúde $(S U S)^{10}$. Além disso, torna possível conhecer a aceitabilidade dos serviços de imunizaçáo e a percepção dos usuários quanto à vacinação, compreender o contexto de baixas coberturas vacinais, as razôes para a não vacinação ou vacinação incompleta, bem como subsidiar e avaliar estratégias locais de inclusão adotadas pelos programas de vacinaçãa ${ }^{10}$.

$\mathrm{O}$ uso de inquéritos domiciliares para o cálculo da $\mathrm{CV}$ real é importante para garantir que os esforços de vacinação sejam eficazes, pois a estimativa de cobertura para cada vacina específica não permite calcular a cobertura pelo esquema completo em cada criança, fornecendo, geralmente, valores superestimados ${ }^{2}$. Além disso, existe uma diferença significativa entre as doses aplicadas e as doses corretas, e os inquéritos muitas vezes revelam divergências em relação aos dados administrativos, assim como problemas de acesso e adesão ao programa ${ }^{2}$. Tendo em vista todas as possibilidades de fragilidade na avaliaçáo da CV realizada por meio de dados de produção é importante que os inquéritos populacionais sejam feitos com maior frequência para que se conheça a real CV de determinada localidade ${ }^{10}$.

Para o MS, a taxa de abandono do programa de vacinaçáo expressa o percentual de crianças que não chegou a completar o número mínimo de doses necessário para se proteger, e tem como fatores mais comuns a falta de esclarecimento, a presença de reaçóes adversas maiores do que as esperadas e o mau atendimento nos serviços de saúde ${ }^{10}$. Portanto, as causas de atraso na realizaçáo das vacinas nos intervalos corretos ou até mesmo a não realização das vacinas devem ser conhecidas pelo serviço de saúde para que a equipe de trabalhadores, principalmente a equipe de enfermagem, tome as devidas providências ${ }^{10}$.

Assim, este trabalho tem por objetivo avaliar a CV real do esquema básico para primeiro ano de vida às crianças maiores de 12 meses e menores de 24 meses numa Unidade de Saúde da Família (USF) de Maceió-AL.

\section{Métodos}

Trata-se de um estudo transversal, realizado nos meses de Janeiro e Fevereiro de 2011. A amostra, do tipo não probabilística, incluiu todas as crianças maiores que 12 e menores que 24 meses cadastradas na USF Dr. Hélvio Auto, cujos responsáveis concordaram em participar da pesquisa. A condição de cadastrado na USF foi confirmada pelo pesquisador mediante a apresentação da certidão de nascimento da criança e/ou documento de identificação com foto do responsável e comparaçáo com ficha de cadastro no momento de recrutamento do indivíduo para participar da pesquisa. Foram excluídas do estudo as crianças cujas carteiras de vacinação não continham informaçôes mínimas para análise, tais como: nome da vacina, número de doses e as datas de administração.

Foi aplicado um instrumento de coleta de dados através de entrevista ao responsável pelas crianças contendo as seguintes variáveis: cobertura do esquema básico completo para as vacinas; doses individuais de cada vacina; idade (meses); sexo; peso ao nascimento; morbidade referida nos últimos três meses; relação com a criança; dados sociodemográficos do responsável (idade, escolaridade e estado civil); idade materna em anos completos; escolaridade materna; classificação econômica da 
família; número de irmãos; posse de seguro-saúde; número de moradores da casa e dificuldade para a não vacinação.

Nosso estudo aplicou o critério de classificação econômica Brasil desenvolvido e publicado pela Associação Brasileira de Empresas de Pesquisa (ABEP, 2007) ${ }^{11}$ para categorizar o indivíduo em uma das 8 prováveis classes econômicas (A1, A2, B1, B2, C1, C2, D, E). Esta categorização obedece a um estudo que atribui pontos à posse de bens e escolaridade do chefe da família ${ }^{11}$.

A carteira de vacinaçáo foi utilizada como fonte de informação. Os dados foram transcritos e quando era detectada alguma situaçấo de atraso em alguma dose de vacina, era realizada convocação imediata e esclarecimentos aos pais ou responsáveis pela criança para atualização da $\mathrm{CV}$.

Considerou-se completado o esquema básico de vacinação e da cobertura de cada vacina quando a criança recebeu a dose das vacinas na idade correta e com intervalos corretos, conforme o método estatístico de análise da $\mathrm{CV}^{2}$.

Depois da análise das carteiras de vacinação, todas as doses de vacinas foram classificadas. Em atraso vacinal (EAV): crianças que, na data do preenchimento, não tinham tomado a vacina adequada para a idade de acordo com as normas nacionais estabelecidas pelo MS. Vacinada com atraso (VCA): crianças vacinadas que receberam a vacina após 15 dias ou mais da data preconizada. Em dia (ED): crianças que receberam a vacina com a idade preconizada ou em até 15 dias após a data referida no calendário de vacinação ou ainda, até 4 dias antes da data preconizada. Vacinada precocemente (VP): crianças que receberam a vacina 5 dias ou mais, antes da data preconizada. Limite excedido (LE): crianças que na data do preenchimento não haviam tomado a vacina e não poderiam recebê-la, em função da idade limite preconizada.

Depois da coleta, os dados foram armazenados em planilha eletrônica (Microsoft Excel $2003^{\circledR}$. Redmond, WA, EUA) na forma de banco de dados. Os resultados foram tabulados e as frequências das variáveis de cada grupo foram calculadas e dispostas nas formas gráfica e tabular.

Os dados tabulados foram processados pelo aplicativo Statistical Package for Social Sciences (SPSS ${ }^{\odot}$ ) (versão 15.0 for Windows, SPSS Inc). A estatística descritiva para as variáveis numéricas incluiu cálculos da média, desvio padrão (DP).

As diferenças nas proporçóes das variáveis entre os grupos de crianças com $\mathrm{CV}$ adequada e náo adequada foram medidas estatisticamente através do Teste do $\chi^{2}$ de Pearson a um nível de significância de $5 \%(\mathrm{p}<0,05)$.

O projeto foi aprovado pelo comitê de Ética em Pesquisa da Universidade Estadual de Ciências da Saúde de Alagoas (Protocolo no 1477).

\section{Resultados}

De um total de 69 crianças maiores de 12 e menores de 24 meses cadastradas na unidade, três não foram localizadas, sendo as 66 demais submetidas à entrevista. As taxas de não resposta a quesitos específicos foram de 7,6\% $(\mathrm{n}=5)$ para peso ao nascer, o qual não constava na caderneta, $1,5 \%(\mathrm{n}=1)$ para idade materna e 3\% $(\mathrm{n}=2)$ para escolaridade materna.

Caracteristicas das crianças: A média de idade foi de $17,6 \pm 3,4$ meses variando entre 12 e 23 meses. A média do peso ao nascer foi de 3238,0 $\pm 469,1$ gramas variando entre 2100 e 4750 gramas. Foi observado que $63,6 \%$ das crianças haviam apresentado algum tipo de morbidade nos três meses que antecederam a entrevista.

Características das famílias: A média de número de moradores foi de $4,7 \pm 1,8$, enquanto que o número médio de irmãos foi de $1,0 \pm 1,0$. Verificou-se que $75,8 \%(\mathrm{n}=50)$ das crianças faziam parte das classes C2 ou D (Tabela 1).

Características do responsável: Em 80,3\% $(\mathrm{n}=53)$ dos casos, a mãe era a responsável pela criança; em $12,1 \%(\mathrm{n}=8)$ os avós; e em 3\% ( $\mathrm{n}=2)$, o pai. Os 4,6\% restantes ficaram igualmente distribuídos para tia, mãe adotiva e babá. A média de idade do responsável foi de 30,53 \pm 11 variando de 16 a 62 anos.

Situação vacinal: Verificou-se que $24 \%(\mathrm{n}=16)$ das crianças completaram o esquema básico de vacinação. A cobertura para as vacinas $\mathrm{HB}$, DTP + Hib, VOP e VORH foram, respectivamente, $72,7 \%(\mathrm{n}=48), 65,2 \%(\mathrm{n}=43), 63,6 \%$ $(\mathrm{n}=42)$ e $77,2 \%(\mathrm{n}=51)$. As porcentagens para cada dose e sua respectiva classificaçấo pode ser vista na Tabela 2 .

Foram realizados cruzamentos estatísticos, no intuito de se estabelecer associaçôes de algumas variáveis e a CV, fato que náo gerou resultados estatisticamente significantes.

\section{Discussão}

As coberturas para cada uma das vacinas foram sempre maiores do que a cobertura para o esquema completo. Observou-se que apenas $24,2 \%$ das crianças estavam com o esquema vacinal completo e que os índices de cobertura de um modo geral foram baixos e inferiores à meta do MS de vacinar 95\% das crianças menores de um ano, exceto em relação a BCG, cuja cobertura preconizada é de $90 \%{ }^{10}$. É importante ressaltar que as vacinas pneumocócica e meningocócica foram introduzidas no calendário nacional em 2011, por isso náo foram registradas na presente pesquisa.

Foram observadas diferenças nas coberturas das vacinas VOP, DTP-Hib e HB, considerando que o esquema básico 
Tabela 1. Esquema básico de vacinação em crianças maiores de 12 e menores de 24 meses de idade segundo idade materna, escolaridade materna, classe econômica, posse de seguro-saúde, dificuldades para a não vacinação, peso ao nascimento, estado civil do responsável, número de irmãos e morbidade referida nos últimos três meses.

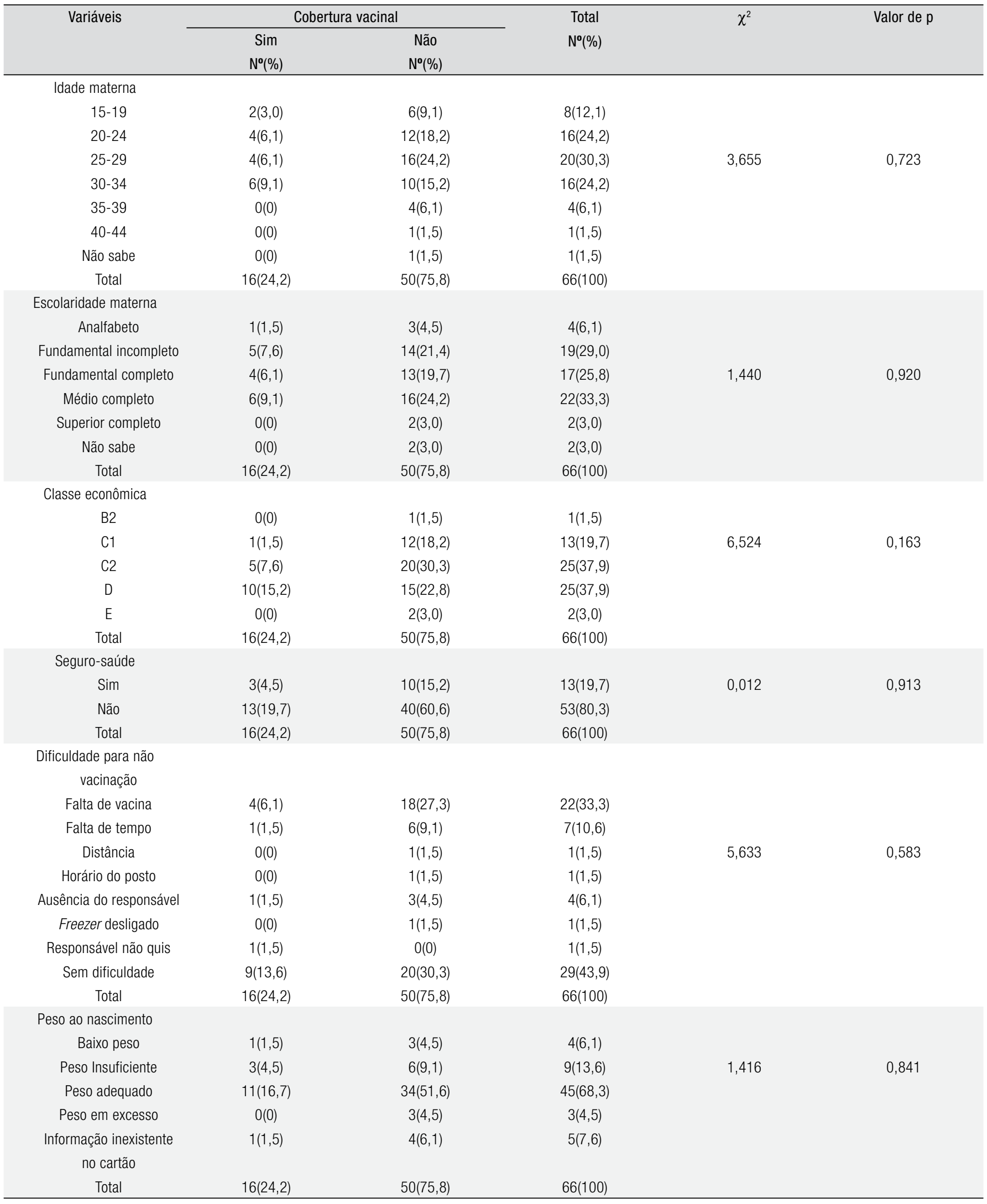

Situação vacinal: $24,2 \%(n=16)$ das crianças completaram 0 esquema básico de vacinação. 


\begin{tabular}{|c|c|c|c|c|c|}
\hline Solteiro & $3(4,5)$ & $13(19,7)$ & $16(24,2)$ & 0,440 & 0,802 \\
\hline Casado & $12(18,2)$ & $35(53,1)$ & $47(71,3)$ & & \\
\hline \multicolumn{6}{|l|}{ Escolaridade do responsável } \\
\hline Analfabeto & $2(3,0)$ & $5(7,6)$ & $7(10,6)$ & & \\
\hline Fundamental Incompleto & $5(7,6)$ & $16(24,2)$ & $21(31,8)$ & 1,161 & 0,885 \\
\hline Fundamental completo & $5(7,6)$ & $13(19,7)$ & $18(27,3)$ & & \\
\hline Médio completo & $4(6,1)$ & $13(19,7)$ & $17(25,8)$ & & \\
\hline 0 & $9(13,6)$ & $15(22,8)$ & $24(36,4)$ & 5,191 & 0,268 \\
\hline 1 & $5(7,6)$ & $16(24,2)$ & $21(31,8)$ & & \\
\hline 2 & $2(3,0)$ & $13(19,7)$ & $15(22,7)$ & & \\
\hline 3 & $0(0)$ & $5(7,6)$ & $5(7,6)$ & & \\
\hline 4 & $0(0)$ & $1(1,5)$ & $1(1,5)$ & & \\
\hline Total & $16(24,2)$ & $50(75,8)$ & $66(100)$ & & \\
\hline \multicolumn{6}{|l|}{ Morbidade } \\
\hline $\operatorname{Sim}$ & $8(12,1)$ & $34(51,6)$ & $42(63,7)$ & 1,697 & 0,193 \\
\hline Não & $8(12,1)$ & $16(24,2)$ & $24(36,4)$ & & \\
\hline Total & $16(24,2)$ & $50(75,8)$ & $66(100)$ & & \\
\hline
\end{tabular}

Situação vacinal: $24,2 \%(n=16)$ das crianças completaram o esquema básico de vacinação.

Tabela 2. Situação vacinal em crianças maiores de 12 e menores de 24 meses de idade de uma Unidade de Saúde da Família, Maceió-AL.

\begin{tabular}{|c|c|c|c|c|c|}
\hline \multirow[t]{3}{*}{ Vacinas } & \multicolumn{5}{|c|}{ Situação vacinal } \\
\hline & ED & VCA & EAV & VP & LE \\
\hline & $\mathrm{N}^{0}(\%)$ & $\mathrm{N}^{0}(\%)$ & $\mathrm{N}^{0}(\%)$ & $\mathrm{N}^{0}(\%)$ & $\mathrm{N}^{\circ}(\%)$ \\
\hline $\mathrm{BCG}$ & $56(84,8)$ & $10(15,2)$ & - & - & - \\
\hline HB1 & $57(86,4)$ & $9(13,6)$ & - & - & - \\
\hline HB2 & $63(95,5)$ & $1(1,5)$ & $1(1,5)$ & $1(1,5)$ & - \\
\hline HB3 & $56(84,8)$ & $7(10,6)$ & $1(1,5)$ & $2(3,0)$ & - \\
\hline DTP+Hib1 & $48(72,7)$ & $16(24,2)$ & $1(1,5)$ & $1(1,5)$ & - \\
\hline DTP+Hib2 & $62(93,9)$ & $2(3,0)$ & $2(3,0)$ & - & - \\
\hline DTP+Hib3 & $56(84,8)$ & $6(9,1)$ & $4(6,1)$ & - & - \\
\hline VOP1 & $49(74,2)$ & $14(21,2)$ & - & $3(4,5)$ & - \\
\hline VOP2 & $61(92,4)$ & $3(4,5)$ & $1(1,5)$ & $1(1,5)$ & - \\
\hline VOP3 & $57(86,4)$ & $5(7,6)$ & $2(3,0)$ & $2(3,0)$ & - \\
\hline SRC & $30(45,5)$ & $18(27,3)$ & $15(22,7)$ & $3(4,5)$ & - \\
\hline VORH1 & $58(87,9)$ & - & - & $4(6,1)$ & $4(6,1)$ \\
\hline VORH2 & $55(83,3)$ & - & - & $2(3,0)$ & $9(13,6)$ \\
\hline
\end{tabular}

Situação ED: Em dia. Situação VCA: Vacinada com atraso. Situação EAV: Em atraso vacinal. Situação VP: Vacinada precocemente. Situação (LE): Limite excedido.

para as três vacinas completam-se aos seis meses de idade e, principalmente, entre as duas primeiras, já que possuem o mesmo intervalo preconizado para a administração das doses. Esse fato pode sugerir de alguma forma, falhas de registro na sala de vacina ou ainda a não realização das vacinas nos intervalos corretos. Essa diferença remete também a possíveis dificuldades do serviço e dos usuários em adequar a aplicação da última dose da vacina contra $\mathrm{HB}$ aos seis meses de idade, juntamente com as últimas doses de pólio e DTP-Hib, uma vez que, o esquema da vacinação contra $\mathrm{HB}$ é diferente, pois a segunda dose deve ser feita com um mês de idade e, havendo atraso na aplicação da segunda dose, o esquema já não será completado aos seis meses de idade, juntamente com pólio e DTP-Hib.

Apesar de o índice de cobertura da BCG ter sido abaixo do preconizado pelo MS, observou-se que ela apresentou o maior índice dentre as demais. Isto sugere uma facilidade na administração, uma vez que é uma vacina aplicada em dose única, não havendo erros quanto ao intervalo entre as doses. Além disso, a vacina é oferecida na maternidade ou mesmo durante sua primeira consulta na UBS 9 . Porém, apesar do maior índice apresentado, o rigor metodológico do presente estudo contribuiu para a obtençáo de um índice menor do que poderia ter sido alcançado.

Em relação à vacina SRC, verificou-se o menor índice de cobertura $(45,5 \%)$ em função de ser administrada nas crianças apenas com um ano de idade, data esta, distante das 
demais doses aplicadas, o que gera um casual esquecimento por parte do responsável ${ }^{12}$.

Não foi observado atraso na administração das doses de VORH. Uma possível explicação pode ser o fato de que houve uma maior orientação na sala de vacina pelo profissional de saúde em relação à idade limite imposta para sua administração: 3 meses e 15 dias para a primeira dose e 5 meses e 15 dias para a segunda dose, não devendo ser aplicada fora desses prazos sob pena de aumentar os riscos de a criança apresentar intussuscepção intestinal, ainda que esses riscos sejam inexpressivos, segundo Simonsen et al. ${ }^{10,12}$.

De acordo com o método de avaliação administrativo fornecido pelo Sistema de Informação da Atenção Básica (SIAB), observou-se que em 2010 a porcentagem de doses aplicadas em crianças com vacinas em dia foi de $82,5 \%$. Comparando-se com o método de avaliação do presente estudo, no qual foi verificada uma taxa de $24,2 \%$ de cobertura vacinal, pode-se constatar uma superestimação nos dados do SIAB. Estas diferenças identificadas nas taxas de cobertura vacinal, entre as duas metodologias de avaliação (administrativa e estatística), devem-se ao fato de que o cálculo da CV do método administrativo, ao ignorar a idade correta para a aplicação, a cobertura calculada a partir de doses aplicadas desconhece a permanência de susceptíveis por mais tempo, inclusive naquelas idades em que o risco de exposição é maior. Ao não considerar os intervalos apropriados entre as doses, a cobertura calculada pode superestimar a proteçáo real, incluindo, entre os vacinados, crianças que, ao receberem as doses com intervalos mais curtos, podem náo ter desenvolvido imunidade ${ }^{2}$.

Os registros na carteira de vacinação indicaram crianças vacinadas com atraso em todas as doses das vacinas, exceto nas doses de VORH. Salientamos que, apesar de ter recebido a vacina e de existir de alguma forma a possibilidade de a criança estar imunizada, mesmo que com atraso, ocorreu durante esse intervalo susceptibilidade para adquirir doenças imunopreveníveis. Orienta-se seguir o mais fielmente possível as recomendaçôes para idade mínima de vacinação e intervalo entre as doses das vacinas ${ }^{8}$. Os esquemas de vacinação não completados não conferem proteção adequada, além de se constituírem num grande desperdício de tempo e de recursos financeiros ${ }^{13}$.

Estavam em maior atraso vacinal as doses de SRC $(22,7 \%)$, DTP + Hib3 (6,1\%), DTP + Hib2 e VOP3 (3\%). As doses de HB2, HB3, DTP + Hib1 e VOP2 apresentaram atraso vacinal de $1,5 \%$. Não houve atraso vacinal nas doses de BCG, HB1, VOP1, e doses de VORH. Isto sugere uma maior preocupaçáo por parte dos pais, no que refere a administração das primeiras doses das vacinas, quando comparado às doses posteriores.
Verificou-se que receberam a dose da vacina precocemente: VORH1 (6,1\%), VOP1 e SRC $(4,5 \%)$, HB3, VOP3 e VORH2 (3\%), HB2 e DTP+Hib1 (1,5\%). Isto revela falta de informação técnica, visto que as doses administradas antes da idade correta são consideradas inválidas, devendo-se revacinar a criança respeitando-se o intervalo mínimo recomendado entre a dose não válida e a nova dose da vacina ${ }^{8}$.

Foi observado que para $56,1 \%$ dos entrevistados houve algum tipo de dificuldade para a não vacinação, dificuldades estas que estão dentre os fatores mais comuns para a taxa de abandono do programa de vacinação de acordo com o MS. Dentre as maiores causas relatadas para a não vacinação estão: falta de vacina na UBS $(33,3 \%)$; ausência do responsável para aplicar a vacina $(6,1 \%)$; falta de tempo $(10,6 \%)$; freezer desligado (1,5\%). Gatti e Oliveira (2005) observaram que $16 \%$ dos entrevistados apontaram o esquecimento como causa da não vacinação, $12 \%$ motivo de doença da criança e horário de funcionamento da UBS ${ }^{14}$. Por sua vez, Ramos et al. (2010) verificaram que a falta de vacina na UBS $(14,3 \%)$ e o esquecimento $(10,7 \%)$ foram as principais dificuldades ${ }^{12}$.

Toda vez que uma pessoa procura um serviço de saúde para receber um imunobiológico e, por algum motivo, não o recebe, caracteriza uma oportunidade perdida em vacinação, muitas vezes relacionada a uma prática dos serviços de saúde ${ }^{15}$. A responsabilidade pela vacinação da populaçáo de uma regiāo pertencente a uma unidade básica não deve se restringir, unicamente, aos componentes da equipe de saúde que aplicam as vacinas; tampouco a vacinação da criança deve ocorrer apenas quando a mãe ou responsável comparece ao serviço para esse fim. Mais do que isso, todos os componentes de uma equipe de saúde devem ser preparados para contribuir, ativamente, para o sucesso de um plano de vacinação e aproveitar todas as oportunidades para verificar e implementar o nível de imunização da população susceptível ${ }^{14}$.

Visto que a CV é um indicador de acesso ao PNI e de efetividade do programa local de imunização, tal fato alerta as equipes dos serviços de saúde do município a fatores, horário e qualidade do atendimento nas salas de vacina, os quais podem interferir no acesso ao programa local ${ }^{10}$. Assim, quanto melhor estruturada estiver a rede de atenção básica, melhores serão o acesso e a adesão ao PNI.

Este trabalho enfatizou a CV real e o perfil das crianças incluídas no estudo. Adicionalmente, foram realizados cruzamentos estatísticos, no intuito de se estabelecer associaçóes de algumas variáveis e a $\mathrm{CV}$, fato que não gerou resultados estatisticamente significantes. Isto evidencia a necessidade de novos estudos com o objetivo de identificar os motivos do atraso em completar o esquema básico.

É imprescindível que as equipes de saúde aprimorem a qualidade do atendimento realizado nas salas de vacinas nas 
UBS, com anotações mais fiéis e cuidado com os relatórios e registros diários. O registro efetivo das vacinas realizadas nas salas de vacina das UBS consiste em estratégia importante, fornece informaçóes fidedignas acerca das doses aplicadas, contribui para o alcance das metas e permite realizar estimativa de retorno das crianças para as próximas doses. As atividades de educação em saúde proporcionam maior conhecimento da população acerca da importância da vacinação e provocam maior envolvimento e compromisso da equipe de saúde na realização desta atividade. A visita rotineira de ACS em todas as residências da área de abrangência consiste em atividade importante para monitorar a situação de saúde das crianças, realizando as orientaçóes de acordo com cada caso, intensificando dessa forma o aumento da $\mathrm{CV}$ e diminuindo os atrasos para completar o esquema básico ${ }^{10}$.

Apesar de a estratégia de atenção primária à saúde ter contribuído decisivamente para o sucesso dos programas de imunização, as unidades de saúde necessitam aprimorar seus procedimentos técnicos e administrativos a fim de garantir a plenitude da CV em data oportuna ${ }^{16}$.

\section{Referências}

1. Miranda AS, Scheibel IM, Tavares MRG, Takeda SMP. Avaliação da cobertura vacinal do esquema básico para o primeiro ano de vida. Rev Saúde Pública. 1995; 29(3):208-14. http://dx.doi.org/10.1590/ S0034-89101995000300008

2. Moraes JC, Ribeiro MCSA, Simões O, Castro PC, Barata RB. Qual a cobertura vacinal real?. Epidemiol Serv Saúde. 2003; 12(3):147-53.

3. Teixeira AMS, Rocha CMV. Vigilância das coberturas de vacinação: uma metodologia para detecção e intervenção em situações de risco. Epidemiol Serv Saúde. 2010; 19(3):217-26. http://dx.doi.org/10.5123/ S1679-49742010000300004

4. Guimarães TMR, Alves JGB, Tavares MMF. Impacto das ações de Imunização pelo Programa Saúde da Família na mortalidade infantil por doenças evitáveis em Olinda, Pernambuco, Brasil. Cad Saúde Pública. 2009; 25(4):868-76. http://dx.doi.org/10.1590/S0102$311 \times 2009000400018$
5. França ISX, Simplício DN, Alves FP, Brito VRS. Cobertura vacinal e mortalidade infantil em Campina Grande, PB, Brasil. Rev Bras Enferm. 2009; 62(2):258-64. http://dx.doi.org/10.1590/S003471672009000200014

6. Morillo SG, Luchs A, Cilli A, Costa FF, Carmona FCC, Timenetsky MCST. Caracterização de genótipos de rotavírus em creches: era pré e pós vacinação contra o rotavirus. J Pediatr. 2010; 86(2). http://dx.doi. org/10.1590/S0021-75572010000200013

7. Brasil. Ministério da Saúde. Secretaria de Vigilância em Saúde. SUS oferece duas novas vacinas para seis milhões de crianças. SVS em rede. 2010; (70) [online] [acesso em 2010 maio 12]. Disponível em: http://189.28.128.179:8080/svs_informa/ edicao-70-fevereiro-de-2010/sus-oferece-duas-novas-vacinas-paraseis-milhoes-de-criancas.

8. Brasil. Ministério da Saúde. Programa Nacional de Vacinação 2006. Brasília: Divisão de Doenças Transmissíveis (DGS), 2006. Orientações técnicas n. 10. Circular Normativa $n^{\circ}$ 08/DT, de 21 de dezembro de 2005.

9. Brasil. Ministério da Saúde. Fundação Nacional de Saúde. Manual de Normas de Vacinação. 4. ed. Brasília: Assessoria de Comunicação e Educação em Saúde; 2001.

10. Pereira DR, Mathias TAF, Soares DFPP, Carvalho WO. Cobertura vacinal em crianças de 12 a 23 meses de idade: estudo exploratório tipo Survey. Rev Eletr Enferm. 2009; 11(2):360-7

11. Associação Brasileira de Empresas de Pesquisa - ABEP. ABEP 2008 Critério de Classificação Econômica Brasil. São Paulo: ABEP; 2007. Disponivel em: http://www.abep.org/default.aspx?usaritem=arquivo s\&iditem $=23$.

12. Ramos CF, Paixão JGM, Danza FCS, Silva AMP, Caçador DF, Dias VDV, et al. Cumprimento do Calendário de vacinação de crianças em uma unidade de saúde da família. Rev Pan-Amaz Saúde 2010; 1(2):55-60.

13. Alagoas. Secretaria de Estado da Saúde - SES/AL. Programa Estadual de Imunização. Cobertura vacinal em Alagoas - análise da situação atual. Superintendente de Vigilância à Saúde; 2007.

14. Gatti MA, Oliveira LR. Crianças faltosas à vacinação condições de vida da família e concepção sobre vacina: um inquérito domiciliar. Salusvita. 2005; 24(3):427-36.

15. Tertuliano GC, Stein AT. Atraso vacinal e seus determinantes: um estudo em localidade atendida pela Estratégia de Saúde da Família. Cien Saude Colet. 2011; 16(2):523-30. http://dx.doi.org/10.1590/ S1413-81232011000200015

16. Molina AC, Godoy I, Carvalho LR, Caldas Junior AL. Situação vacinal infantil e características individuais e familiares do interior de São Paulo. Acta Sci Health Sci. 2007; 29(2):99-106. 